\title{
Simple analytic model for subduction zone thermal structure
}

\author{
J. Huw Davies \\ Department of Earth Sciences, The University of Liverpool, Brownlow Street, Liverpool, L69 3BX, UK. E-mail: davies@liv.ac.uk
}

Accepted 1999 July 7. Received 1999 July 2; in original form 1998 October 23

\begin{abstract}
SUMMAR Y
A new analytic model is presented for the thermal structure of subduction zones. It applies to the deeper regions of a subduction zone, where the overriding mantle is no longer rigid but flows parallel to the slab surface. The model captures the development of one thermal boundary layer out into the mantle wedge, and another into the subducting slab. By combining this model with the analytic model of Royden (1993a,b), which applies to regions in which the overriding plate is rigid, a nearly complete analytic model for the thermal structure of a steady-state subduction zone can be achieved. A good agreement is demonstrated between the output of the combined analytic model and a numerical finite element calculation. The advantages of this analytic approach include (1) efficiency (only limited computing resources are needed); (2) flexibility (non-linear slab shape, and processes such as erosion, and shear heating are easily incorporated); and (3) transparency (the effect of changes in input variables can be seen directly).
\end{abstract}

Key words: analytic model, subduction thermal structure, subduction zone.

\section{INTRODUCTION}

Thermal structures of subduction zones are important for understanding many observations, including subduction zone magmatism, surface heat flow, gravity signature, seismic velocity, seismic attenuation, seismicity and metamorphism, and for estimating the rheology and mineralogy of subduction zones.

Numerical methods (finite difference and finite element) have been used to calculate the thermal structure of subduction zones for three decades (e.g. Toksöz et al. 1971; Honda 1985; Davies \& Stevenson 1992). These methods can in principle (1) cope with a wide range of geometries and boundary conditions; (2) provide a solution throughout the model domain; and (3) model the time evolution. Their major disadvantage is that they can be expensive to develop, set up and calculate. For example numerical calculations are very time-consuming to set up if realistic slab shapes are to be incorporated. There have been very few attempts to generate thermal models of subduction zones with an angle of dip other than constant. These include that by Ponko \& Peacock (1995), who modelled the shape of a subduction zone by approximating it by two straight line segments; and that by van den Beukel (1990) and co-workers, who modelled a subducting slab as an arc of a circle. If one needs to model many subduction zones at a fine resolution incorporating realistic slab shapes, then utilizing analytic models is the only way presently available. Another advantage of an analytic model is infinite resolution, so integrals of properties dependent on temperature (e.g. total lithospheric strength) can be evaluated to a constrained error. Analytic models also allow one to see directly the impact of changing the input parameters.

The first analytic solution to the thermal structure of a subduction zone was by McKenzie (1969). He assumed subduction of lithosphere into a constant-temperature mantle. This model illustrated how the slab would heat up with time, but did not include the effect of the cooling of the mantle by the subducting slab. Davies \& Stevenson (1992, Appendix A) presented a solution where the subducting lithosphere cooled the mantle, as well as allowing the mantle to heat the slab. In this model it was assumed that the mantle flow was parallel and equal to the slab velocity, and that the slab had an initial linear thermal gradient. Molnar \& England (1990) presented an analytic solution for the temperature on the interplate thrust (i.e. where the overriding plate is rigid), derived by equating heat fluxes across the thrust. Royden (1993a,b) extended this to allow the solution for the temperature structure for both the downgoing plate and the overriding rigid lithosphere, assuming no lateral heat conduction (which holds well for low angles of dip). Her solution allowed the incorporation of shear and radioactive heating. One can also prescribe accretion (or erosion) of material to the downgoing plate, and erosion of (or deposition at) the surface of the overriding plate. Steady state is assumed in all the analytic solutions in the reference frame fixed to the overriding plate. 
In this paper, I will first introduce an improvement to the model of Davies \& Stevenson (1992), which allows for an initial thermal boundary layer in the mantle wedge as well as in the slab. I then show how this model can be combined with the model of Royden (1993a) to provide a flexible tool to model the thermal structure of subduction zones. Finally, I compare two similar models, one produced numerically, and the other by an appropriate combination of analytic models.

\section{NEW ANALYTIC MODEL}

The analytic model assumes (1) that the mantle above the slab flows parallel to the subducting plate and at the same velocity, and (2) that the conduction down the slab dip can be ignored. If the mantle wedge is well coupled to the subducting slab, then the first assumption is a good approximation at least close to the slab surface. If the thermal gradient perpendicular to the slab surface is much greater than the thermal gradient down the slab dip, then the second assumption is also a good approximation.

The geometry and co-ordinate axes for the model are defined in Fig. 1(a), and the temperature at $y=0$ is defined in Fig. 1(b). The $x$-axis is perpendicular to the slab surface, with the positive direction being into the subducting plate, and the positive $y$-axis is in the down-dip direction. The origins of both axes are on the top surface of the subducting plate at the shallowest depth for the calculation. The sensible shallowest depth for the model is the point in the mantle at which the asthenosphere flow in the overriding mantle wedge first becomes parallel
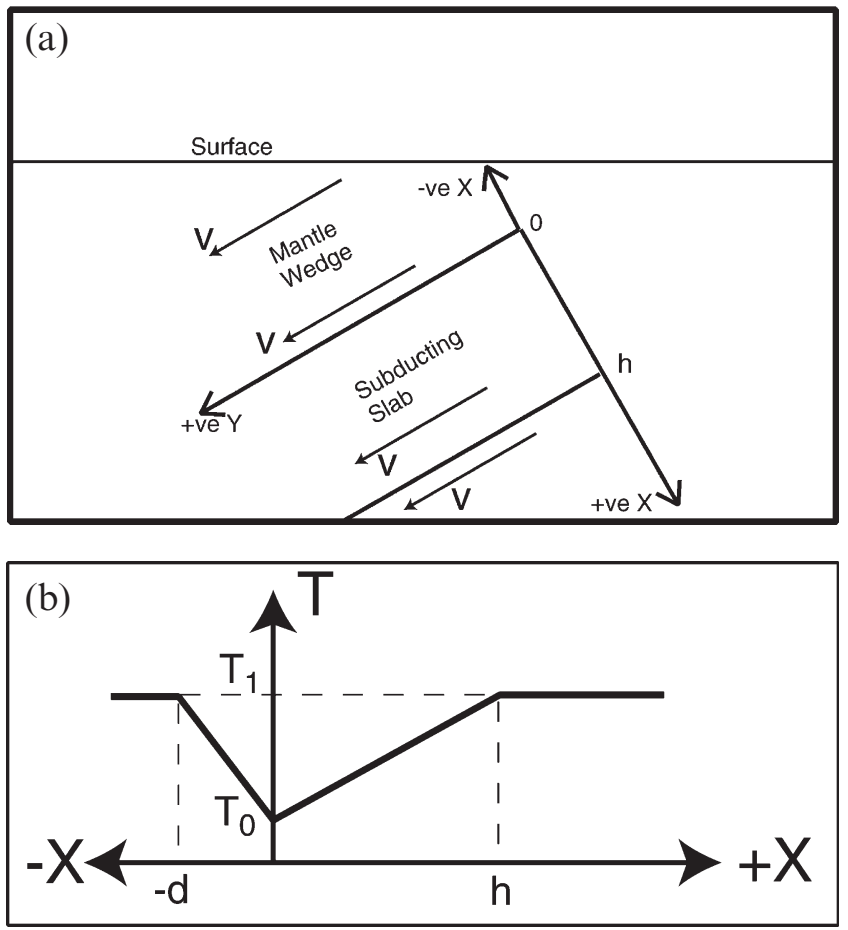

Figure 1. (a) The geometry, orientation and co-ordinate system of the analytic thermal model. Negative $x$ is out into the overriding mantle wedge, and positive $x$ is into the slab. $x=0$ is the upper surface of the slab. (b) The initial temperature at $y=0$, i.e. the temperature of the slab as it subducts into the asthenosphere. to the subducting slab. This will be some tens of kilometres greater than the thickness of the mechanical lithosphere, and can be expected to vary with the convergence velocity.

The boundary conditions assumed are as follows.

For $y=0$ (Fig. 1b),

$T=T_{1} \quad$ for $x \leq-d$,

$T=T_{0}+\left(\frac{-x}{d}\right)\left(T_{1}-T_{0}\right) \quad$ for $-d \leq x \leq 0$,

$T=T_{0}+\left(\frac{x}{h}\right)\left(T_{1}-T_{0}\right) \quad$ for $0 \leq x \leq h$,

$T=T_{1} \quad$ for $x \geq h$.

As $y \rightarrow \infty, \quad T($ all $x) \rightarrow T_{1}$,

As $x \rightarrow \infty, \quad T($ all $y) \rightarrow T_{1}$.

Eq. (1a) implies that the minimum temperature at the thrust is $T_{0}$, while the mantle temperature away from the subduction zone is assumed to be $T_{1}$. The initial width of the thermal anomaly in the mantle wedge is $d$, while the thickness of the subducting thermal lithosphere is assumed to be $h$. Eqs 1(b) and 1(c) follow, since a steady-state solution is assumed. The assumption of constant temperature $\left(T_{1}\right)$ with depth through the mantle far away from the subduction zone is valid if one is solving for potential temperature in a vigorously convecting asthenosphere. One can convert from potential temperature to actual temperature by adding the appropriate adiabatic gradient. Both the diffusivity $\kappa$, and the subduction velocity $v$ are assumed to be constant throughout the solution domain.

The heat transfer equation is

$\frac{\partial T}{\partial t}+\mathbf{v} \cdot \nabla T=\kappa \nabla^{2} T+\frac{Q}{\rho C_{\mathrm{p}}}$,

where $T$ is temperature, $t$ is time, $\mathbf{v}$ is the velocity vector, $\kappa$ is thermal diffusivity, $Q$ is any volumetric heat source or sink, $\rho$ is density, and $C_{\mathrm{p}}$ is specific heat capacity. If we assume a steady state $(\partial T / \partial t=0)$, a reference frame attached to the overriding plate (such that $\mathbf{v}$ is the convergence velocity), no heat sources or sinks $(Q=0)$, that the subduction zone extends infinitely in the $z$-(along arc) direction (so $\partial T / \partial z=0$, etc.), that the velocity is parallel to the $y$-axis $\left(v_{x}=0\right)$, and that the downdip temperature gradients are much lower than the gradients perpendicular to the slab surface $\left(\partial^{2} T / \partial y^{2} \ll \partial^{2} T / \partial x^{2}\right)$ then eq. (2) reduces to

$\mathbf{v}_{y} \frac{\partial T}{\partial y}=\kappa \frac{\partial^{2} T}{\partial x^{2}}$.

Adapting a solution from Carslaw \& Jaeger (1959, eq. 1, p. 53), by replacing $t$ with $y / v$, it can be shown that

$T=\frac{1}{2 \sqrt{\pi y \kappa / v}} \int_{-\infty}^{\infty} f\left(x^{\prime}\right) \exp \left[-\left(x-x^{\prime}\right)^{2} /(4 y \kappa / v)\right] d x^{\prime}$

is the solution of eq. (3), where $f(x)$ is the input boundary condition (i.e. the temperature at $y=0)$. With $f(x)$ as displayed in Fig. 1(b) and defined in eq. (1a), the integral in eq. (4) 
(a) $\quad \mathrm{X}$-axis (across-slab distance $[\mathrm{km}])$

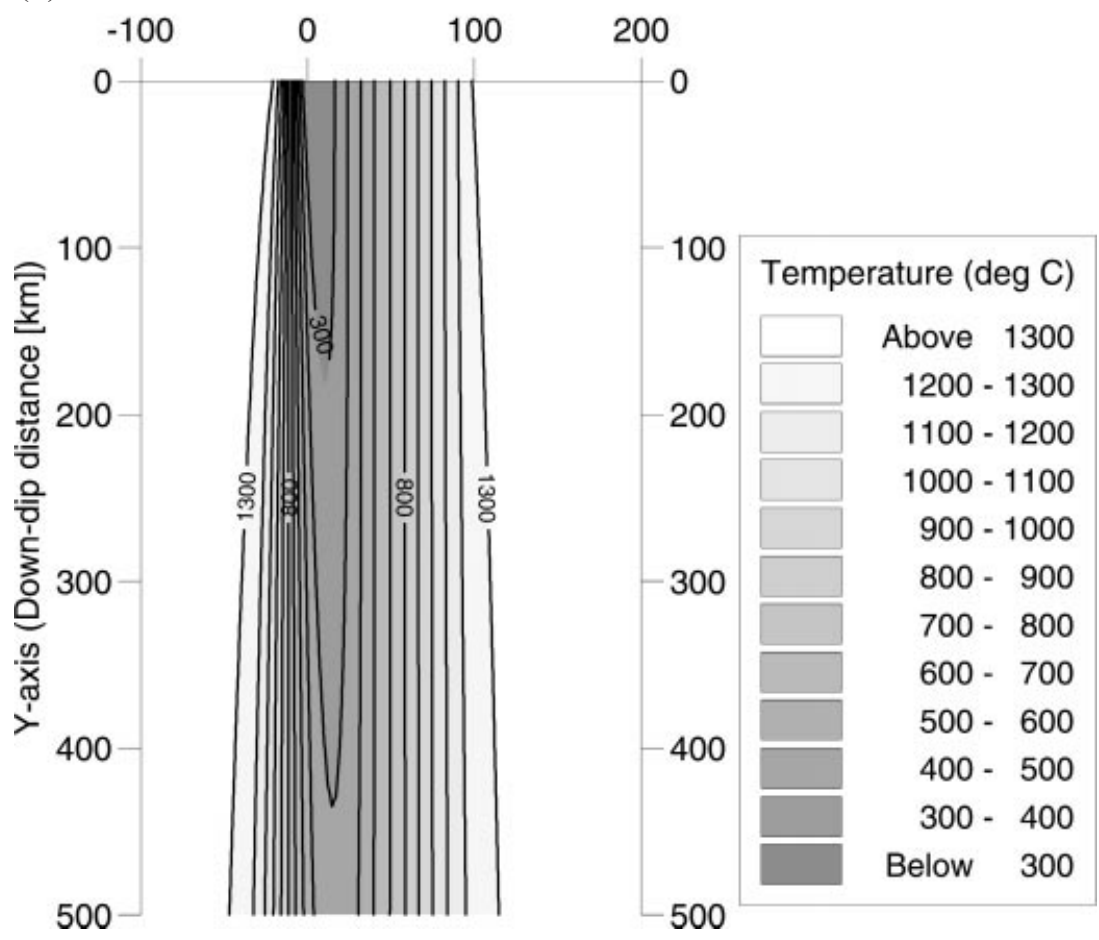

(b) X-axis (across-slab distance $[\mathrm{km}]$ )

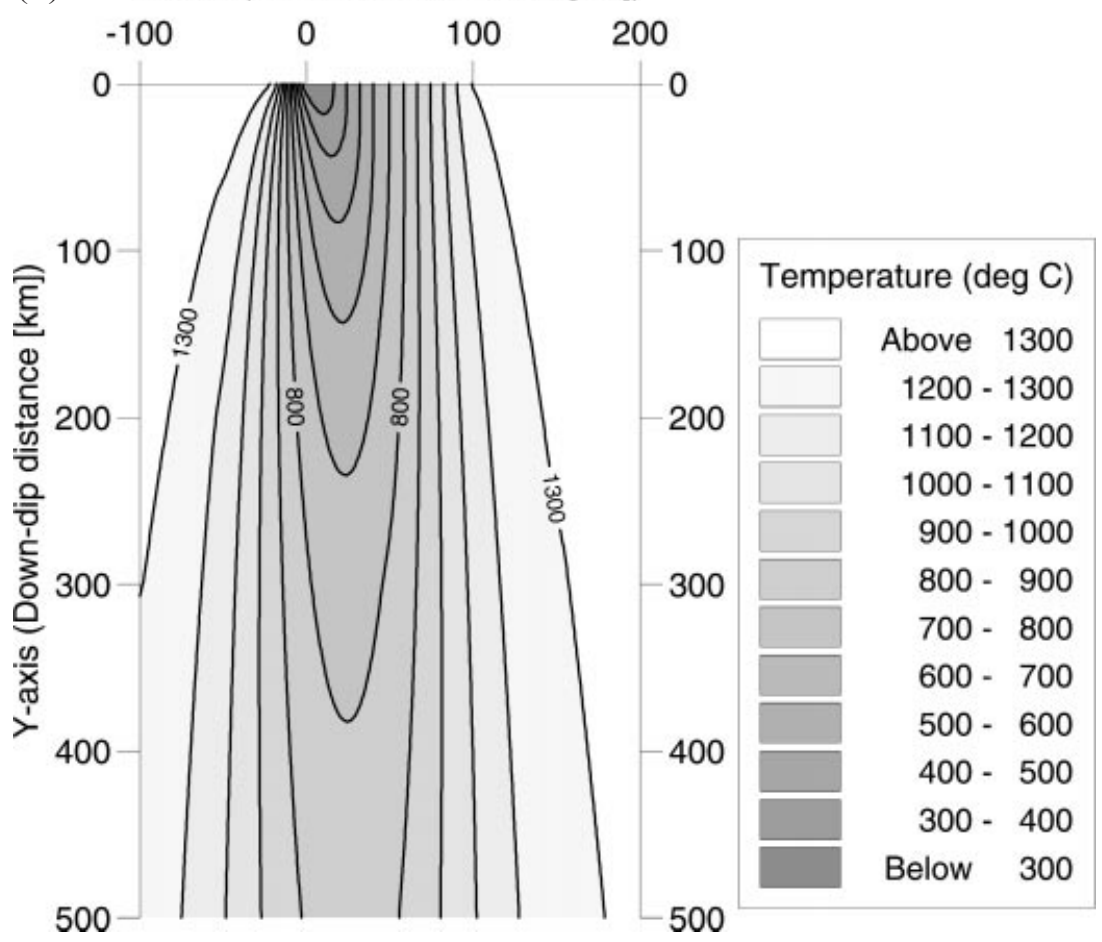

Figure 2. Solutions for the analytic model (eq. 4). It is assumed that the lithosphere thickness $h=100 \mathrm{~km}$; the initial thickness of the boundary layer in the mantle wedge $d=20 \mathrm{~km}$; the thermal diffusivity $\kappa=10^{-6} \mathrm{~m}^{2} \mathrm{~s}^{-1}$; the mantle temperature $T_{1}=1325^{\circ} \mathrm{C}$; the initial minimum temperature on the thrust $T_{0}=100^{\circ} \mathrm{C}$; and that the subducting velocity, $\mathbf{v}$, is (a) $10 \mathrm{~cm} \mathrm{yr}^{-1}$ (b) $1 \mathrm{~cm} \mathrm{yr}^{-1}$. Note that the solution illustrates not only the heating of the subducting slab with distance down dip, but also the cooling of the mantle wedge. At slower subduction velocities, the heating of the slab and the cooling of the wedge are more pronounced at shallower depths. The horizontal axis is the $x$-axis (i.e. across the slab), while the vertical direction is in the y-direction (i.e. down dip). 
reduces to

$$
\begin{aligned}
T= & T_{1}+\left(\frac{T_{1}-T_{0}}{h}\right)\left\{\left(\frac{x-h}{2}\right)[\operatorname{erf}(-b)-\operatorname{erf}(-a)]\right. \\
& \left.-\left(\sqrt{\frac{\kappa y}{v \pi}}\right)\left[\exp \left(-b^{2}\right)-\exp \left(-a^{2}\right)\right]\right\} \\
& +\left(\frac{T_{1}-T_{0}}{d}\right)\left\{\left(\frac{x+d}{2}\right)[\operatorname{erf}(-e)-\operatorname{erf}(-f)]\right. \\
& \left.-\left(\sqrt{\frac{\kappa y}{v \pi}}\right)\left[\exp \left(-e^{2}\right)-\exp \left(-f^{2}\right)\right]\right\},
\end{aligned}
$$

where

$$
\begin{array}{ll}
a=\frac{x}{\sqrt{4 y \kappa / v}}, & b=\frac{x-h}{\sqrt{4 y \kappa / v}}, \\
e=\frac{x+d}{\sqrt{4 y \kappa / v}}, & f=\frac{x}{\sqrt{4 y \kappa / v}}=a,
\end{array}
$$

and erf is the error function, which can be approximated by polynomials to desired levels of accuracy (Abramowitz \& Stegun (1964). Fig. 2(a) shows the solution for the case $\mathbf{v}=10 \mathrm{~cm} \mathrm{yr}^{-1}$, while Fig. 2(b) is for $\mathbf{v}=1 \mathrm{~cm} \mathrm{yr}^{-1}$. Note that the models show cooling of the wedge and heating of the slab with depth. The cooling and heating are more pronounced at shallower depths for slower convergence velocities. From the analytic solution one can see that, apart from $d$ and $h$ (and the trivial $T_{0}$ and $\left.T_{1}\right)$, the only other variable is the combination $(\kappa y / v)^{1 / 2}$. Hence a single result (e.g. Fig. 2a) can be used to provide the result for all $\kappa$ and $v$, by just varying the distance down dip $(y)$ at which the temperature is evaluated. For example, Fig. 2(b) could be obtained directly from Fig. 2(a) just by reducing the scale in the down-dip direction by a factor of 10 .

If one lets $y$ be the accumulated distance down dip along the slab surface, while one considers $x$ to be the distance locally perpendicular to the slab surface, then one can trivially extend the model to a slab with variable dip down its length (see Fig. 3). We do not demonstrate it rigorously, but one can

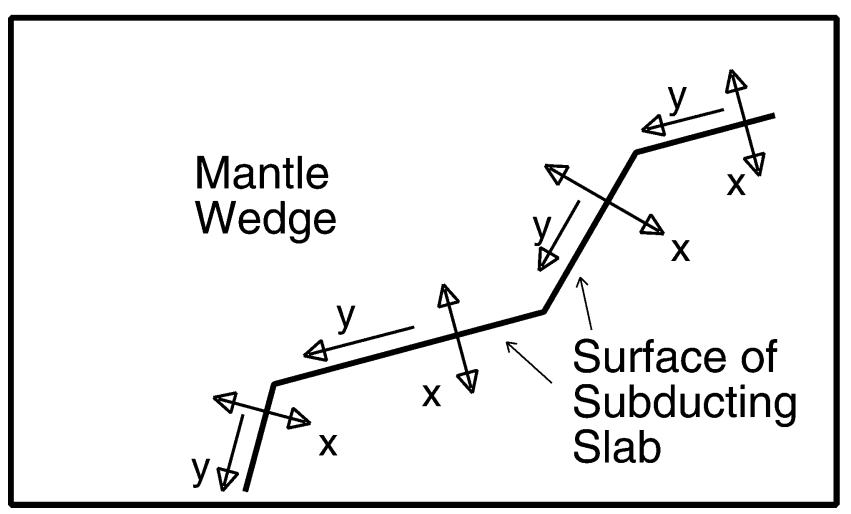

Figure 3. Illustration of how the calculation might be trivially adapted to solve for temperatures in curved subducting slabs. Note that $y$ is defined as the distance down dip, while $x$ is the perpendicular distance from the slab surface at that point. This adaptation can be expected to be good provided that the slab curvature is low. It allows the analytic model to be used to obtain approximate thermal structures for cases with varying slab shape. expect this simple adaptation to produce reasonable results provided the fundamental assumptions still hold. Since one can expect the mantle wedge flow near the slab surface to remain parallel to the surface of a slab with varying dip, and the conduction to remain dominantly perpendicular to the surface, the underlying assumptions of the analytic model will remain valid. Away from the slab surface $(|x| \gg 0)$, in regions of slab curvature, this adaptation stretches or compresses the thermal field in the down-dip direction. Provided the downdip thermal gradient is small (a required assumption of the basic model), this is not a problem. So provided the slab curvature is not too high, this adaptation should allow the analytic model to be applied to realistic slab shapes. This adaptation could also be applied to numerical models. This simple adaptation is easy to implement with this analytic model since the co-ordinate system of the calculation is tied to the subducting slab. As pointed out earlier, the extra effort in incorporating realistic slab shapes in numerical thermal models is demonstrated by the fact that all subduction zone thermal models published to date involve either simple approximations to slab shapes or even cruder constant slab dip.

\section{COMBINING ANALYTIC MODELS}

One can produce an analytic model for the thermal field of a whole subduction zone. This is done by combining the model of Royden (1993a,b) for the shallow region, where the overriding plate is rigid, with the model described above for the deeper region, where the surrounding mantle is fluid. In Fig. 4 we show the domains in which the various calculations were undertaken. In regions 3 and 4 the linear gradient is from a mantle potential temperature at $100 \mathrm{~km}$ depth to zero at the surface. In regions 5 and 6 the temperature was set to the mantle potential temperature. The temperature along the thrust

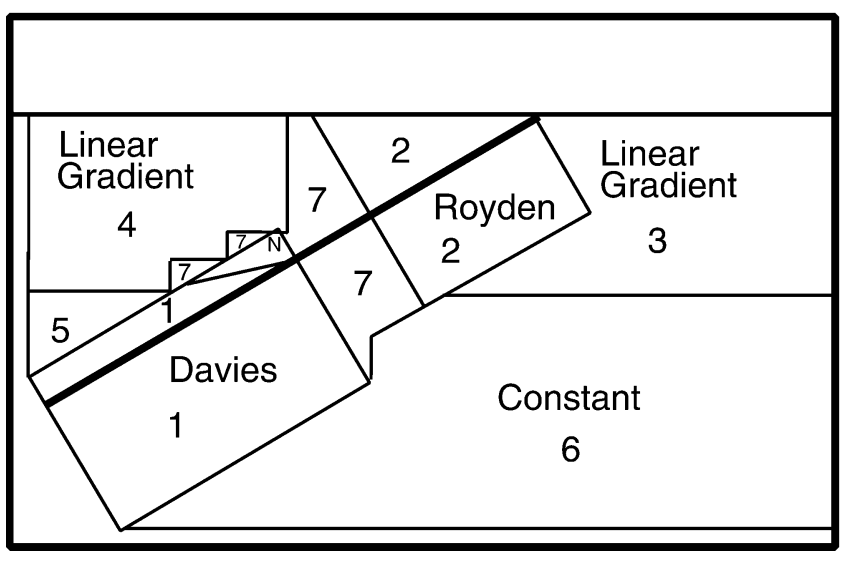

Figure 4. Illustration of the various domains that are used to calculate the thermal structure of the complete subduction zone analytically. In region 1 the model introduced in this paper is used. The small region labelled $\mathrm{N}$ is ignored since the boundary condition of mantle temperature is clearly unreasonable. The model of Royden $(1993 \mathrm{a}, \mathrm{b})$ is used in region 2. In regions 3 and 4 a linear gradient is input, while in regions 5 and 6 a constant temperature is input. No calculation is undertaken in region 7 . The heavy line is the mega-thrust. The temperature is extrapolated along the mega-thrust from region 2 to region 1 . This is done using the same increase of temperature with depth along the thrust as evaluated in region 2. 
(a)

$\mathrm{km}$

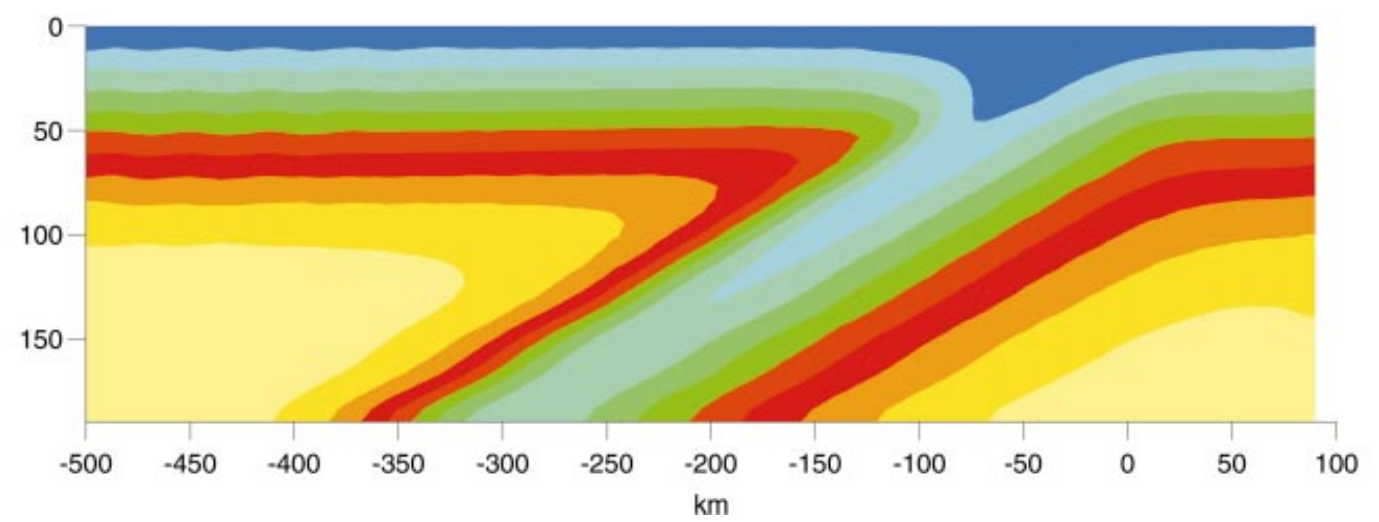

\begin{tabular}{|r|r|}
\hline & Above 1350 \\
$1200-1350$ \\
$1050-1200$ \\
\hline $900-1050$ \\
$750-900$ \\
$600-750$ \\
$450-600$ \\
$300-450$ \\
\hline $150-300$ \\
\hline Below 150 \\
\hline$\square$
\end{tabular}

(b)
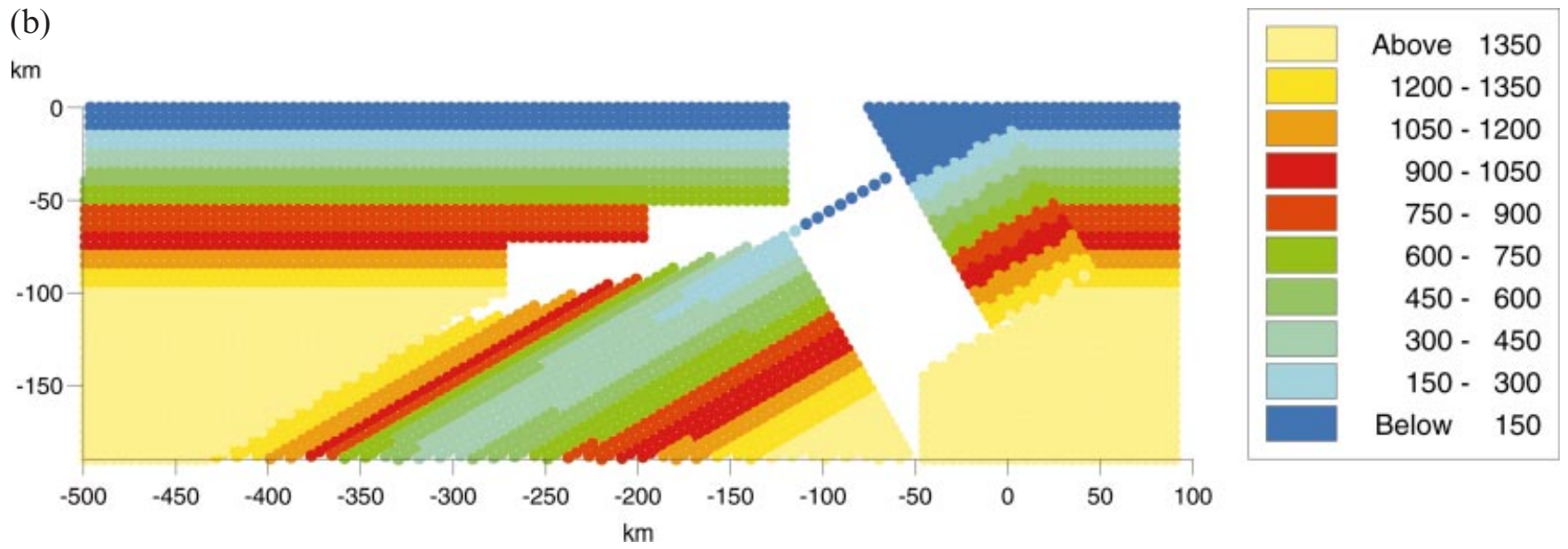

(c)
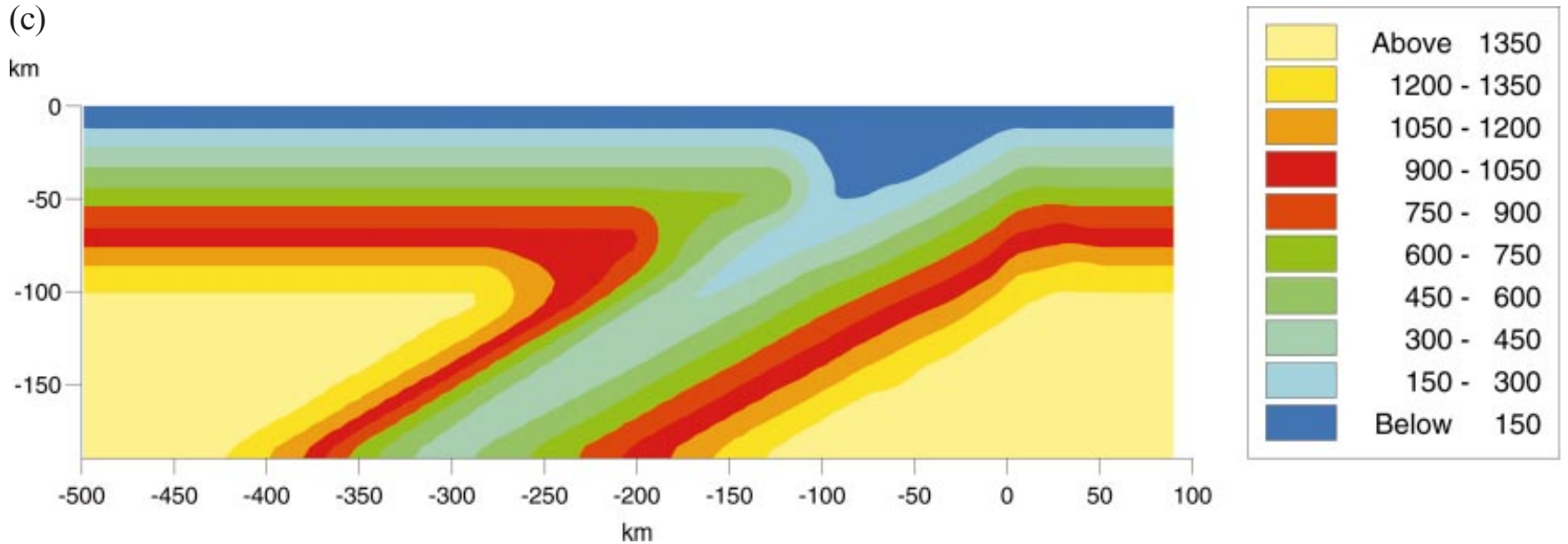

Figure 5. (a) Thermal structure, in ${ }^{\circ} \mathrm{C}$, derived from a numerical model for a subducting slab with a dip of $30^{\circ}$ (after Davies $\&$ Stevenson 1992 ). (b) The actual temperature values calculated analytically for a problem similar to (a); (c) final thermal structure achieved from (b), using simple bilinear interpolation to obtain values in region 7 of Fig. 4 (the empty region in (b) where no calculations were undertaken). Note that (c) is very similar to (a), illustrating that this approach can achieve good accuracy.

(the heavy line in Fig. 4), from the end of the Royden calculation to the start of the Davies calculation, was calculated assuming a linear extrapolation in depth of the deepest thrust temperature from the Royden model. After the calculation, the adiabatic gradient was added throughout to the potential temperatures calculated in the analytic model. This was to facilitate comparison with the results of a finite element numerical model that incorporated the adiabatic gradient into its boundary conditions, and hence was solving for actual, not potential temperatures. 


\section{COMPARISON OF NUMERICAL AND ANALYTIC MODELS}

In Fig. 5(a), I show the temperature of the $30^{\circ}$ dip model from Davies \& Stevenson (1992) solved using a finite element program, with an error function boundary condition on the oceanic lithosphere and a linear geotherm for the continental lithosphere. The thickness of the overriding mechanical lithosphere is $40 \mathrm{~km}$, while the thickness of the thermal lithosphere is $100 \mathrm{~km}$. I have converted the results of both models to dimensional temperatures, assuming that the temperature at the base of the lithosphere is $1350^{\circ} \mathrm{C}$, while the temperature at the surface is assumed to be $0{ }^{\circ} \mathrm{C}$.

A combined analytic model was produced for similar parameters. The only free parameters are effectively the thickness of the thermal boundary layer above the subducting slab $(d)$, and the depth at which the new model is initiated. I used a value of $20 \mathrm{~km}$ for the thickness of the boundary layer and assumed that the origin of the calculation was at $70 \mathrm{~km}$ depth.

As can be seen from Fig. 5(b), the analytic model results do not define the temperature everywhere, but if one interpolates using bilinear interpolation into the areas without values, a final picture can be produced (Fig. 5c) which can be compared directly with the numerical model results (Fig. 5a). The differences between Fig. 5(c) and the numerical model in Fig. 5(a) are very small, and are largest in the wedge corner, which neither the Davies nor Royden analytic model captures. Some differences result from the different boundary conditions on the subducting oceanic crust, namely linear (analytic model) versus error function (numerical model). Given that the difference in computational cost is very large, around a thousandfold, the similarity in the comparison is striking. There is also a large difference in the programming of the two methods, and in setting up runs with variable geometry. The analytic model could be further improved, if desired, by calibrating the thickness of the boundary layer and the origin of the calculation using a suite of numerical models.

This computationally efficient tool has already been used to thermally model cross-sections every $50 \mathrm{~km}$ along-strike for all the world's major subduction zones, incorporating realistic slab shapes (Rhodes 1998). Such a model incorporating the actual convergence velocities and oceanic ages was undertaken to generate an a priori seismic model to improve earthquake relocation in global body-wave tomography.

\section{CONCLUSIONS}

I have presented a new analytic thermal model to allow calculations for the temperature in the mantle wedge and subducting slab. I have shown how it can be combined with results from the sophisticated analytic thermal model of Royden (1993a,b) to allow extremely efficient thermal modelling throughout a subduction zone, that is in both the thrust and mantle wedge region. Such a model is very flexible, and is very efficient computationally compared with numerical models. Provided that the assumptions on which the model is based are valid, the results of the analytic model have been shown to compare favourably with the results of a numerical model. It is likely that the errors introduced from utilizing the analytic model rather than a numerical method are less than the inherent errors arising from uncertainties in model assumptions and parameter values.

\section{ACKNOWLEDGMENTS}

I would like to acknowledge Brad Hager for initially suggesting an analytic model, the interaction with Mark Rhodes and Andrea Rowland, an informal review of an earlier draft by Nick Kusznir, and constructive reviews from Leigh Royden and Paul Morgan.

\section{REFERENCES}

Abramowitz, M. \& Stegun, I., eds, 1964. Handbook of Mathematical Functions, National Bureau of Standards, Washington, DC.

Carslaw, H.S. \& Jaeger, J.C., 1959. Conduction of Heat in Solids, Clarendon, Oxford.

Davies, J.H. \& Stevenson, D.J., 1992. Physical model for the source region of subduction zone volcanics, J. geophys. Res., 97, 2037-2070.

Honda, S., 1985. Thermal structure beneath Tohoku, northeast JapanA case study for understanding the detailed thermal structure of the subduction zone, Tectonophysics, 112, 69-102.

McKenzie, D.P., 1969. Speculations on the consequences and causes of plate motions, Geophys. J. R. astr. Soc., 18, 1-32.

Molnar, P. \& England, P.C., 1990. Temperatures, heat flux, and frictional stress near major thrust faults, J. geophys. Res., 95, 4833-4856.

Ponko, S.C. \& Peacock, S.M., 1995. Thermal modelling of the Alaska subduction zone: Insight into the petrology of the subducting slab and overlying wedge, J. geophys. Res., 100, 22 117-22 128.

Rhodes, M., 1998. Mantle seismic tomography using P-wave travel times and a priori velocity models, $P h D$ thesis, University of Liverpool.

Royden, L.H., 1993a. The steady state thermal structure of eroding orogenic belts and accretionary prisms, J. geophys. Res., 98, 4487-4507.

Royden, L.H., 1993b. Correction to 'The steady state thermal structure of eroding orogenic belts and accretionary prisms', J. geophys. Res., 98, 20039.

Toksöz, M.N., Minear, J.W. \& Julian, B.R., 1971. Temperature field and geophysical effects of a downgoing slab, J. geophys. Res., 76, $1113-1138$

van den Beukel, J., 1980. Thermal and mechanical modelling of convergent plate margins, $P h D$ thesis, University of Utrecht. 